\title{
Impact(s) des technologies numériques en période de crise : cas du conflit à Mango, Togo
}

The use of technologies by the youth in crisis: the case of conflict with mango, Togo

Impacto (s) de las tecnologías digitales en tiempos de crisis: caso del conflicto en mango, Togo

\section{Namoin Yao}

\section{OpenEdition}

\section{Journals}

Édition électronique

URL : http://journals.openedition.org/ctd/574

DOI : $10.4000 /$ ctd. 574

ISSN : 2491-1437

Éditeur

Chaire Unesco Pratiques émergentes en technologies et communication pour le développement

Référence électronique

Namoin Yao, «Impact(s) des technologies numériques en période de crise : cas du conflit à Mango, Togo », Communication, technologies et développement [En ligne], 6 | 2018, mis en ligne le 04 septembre 2017, consulté le 10 décembre 2020. URL : http://journals.openedition.org/ctd/574 ; DOI : https:// doi.org/10.4000/ctd. 574

Ce document a été généré automatiquement le 10 décembre 2020.

Communication, technologies et développement 


\section{Impact(s) des technologies numériques en période de crise : cas du conflit à Mango, Togo}

The use of technologies by the youth in crisis: the case of conflict with mango, Togo Impacto (s) de las tecnologías digitales en tiempos de crisis: caso del conflicto en mango, Togo

\section{Namoin Yao}

Ces dernières décennies, les conflits/crises sont devenus des éléments de l'ère contemporaine au regard de l'actualité médiatique. En effet, le développement fulgurant des moyens de communication a donné une dimension planétaire à l'information et celle relative aux conflits/ crises n'est pas restée en marge (construction médiatique). N'Dimina Mougala (2007) note à ce propos une recrudescence des crises/conflits en Afrique depuis les années 1998.

Plusieurs publications académiques ont analysé et critiqué le rôle des médias lors des conflits sur le continent (Frère, 2005). Il n'en demeure pas moins qu'ils jouent un rôle de premier plan en informant l'opinion publique. La démocratisation des usages rendue possible grâce à l'accessibilité des outils (Internet et téléphones mobiles) a mis à jour de nouvelles logiques de production d'informations (Champion, 2012). Bon nombre de travaux ont dénoncé les dérives du journalisme à l'ère du web 2.0 où tout le monde peut devenir « journaliste» en publiant des données (Serres, 2010). Nonobstant, ces pratiques constituent pour les citoyens une manière de produire de l'information sur les sujets qui les intéressent ${ }^{1}$.

3 Au Togo, au cours de l'année 2015, un conflit social a surgi dans la ville de Mango ${ }^{2}$. Celuici opposait les autorités locales à une partie de la population à cause de la (ré) installation d'une aire protégée sur une partie du territoire. Ce conflit qui fera par la suite des victimes aussi bien du côté des autorités locales que de la population a été couvert par les médias nationaux et internationaux. Les populations riveraines ainsi que les natifs de la 
préfecture de l'Oti résidant à Lomé ont également produit et diffusé de l'information durant cette période.

4 Les articles publiés (notamment dans la presse écrite ${ }^{3}$ ) ont mis l'accent sur la mobilisation des « filles et fils du milieu résidant à Lomé ». Lors des reportages télévisés, ces derniers affirment être informés en temps réel par «leurs frères » vivant à Mango. Le discours médiatique et la narration de ce conflit ont mis en exergue plusieurs facteurs explicatifs du conflit tels que le déficit de communication (de la part des pouvoirs politiques nationaux et locaux) et la mise à l'écart des populations riveraines pour une exécution efficiente de ce projet. Ils ont également relevé une mobilisation des jeunes natifs du milieu résidant sur place et à Lomé pour la résolution de ce conflit ${ }^{4}$. De ce fait, la lecture de conflit laissait entrevoir un schéma où les populations étaient impliquées ici (Lomé la capitale) et là-bas (ville de Mango). De quelle manière, cette implication a- t-elle été possible? Quels ont été le rôle et l'impact de technologies numériques dans ce conflit? Combien de temps a-t-il duré et comment a-t-il évolué ? Que révèle la gestion de cette crise sur l'état de la communication publique dans ce pays?

Pour y répondre, nous émettons l'hypothèse principale selon laquelle, les populations riveraines et les natifs de la localité résidant à Lomé ont eu davantage recours aux Technologies de l'Information et de la Communication (TIC) qu'aux médias traditionnels pour informer l'opinion publique sur le projet gouvernemental de réinstallation des aires protégées et l'opposition y afférente. Comme hypothèse secondaire, nous postulons que ces usages ont eu impact sur le conflit au regard des décisions prises par les pouvoirs politiques en aval. Cependant, cet impact est à relativiser puisque les technologies n'ont pas contribué à la résolution définitive du conflit ${ }^{5}$.

6 Dans le cadre de cet appel à articles de Communication, Technologies et Développement portant sur « Technologies numériques et diffusion de l'information durant les périodes de conflit et de crise dans le monde ", nous souhaitons nous intéresser à la manière dont ces populations ont eu recours aux technologies d'une part, et à leur impact sur la résolution de ce conflit d'autre part.

7 Sur le plan méthodologique et d'un point de vue théorique cette recherche s'inscrit en Sciences de l'Information et de la Communication en convoquant des travaux sur l'(es) usage (s) des TIC en période de crises/conflits (Proulx, 2015 ; Tine et Elhaou, 2015). Étant donné la nature du conflit qui est l'opposition à un projet gouvernemental, des travaux sur la communication publique seront également convoqués. Enfin, sans nous inscrire dans l'approche déterministe des technologies, nous ancrons notre réflexion dans le cadre de cette recherche aux travaux de Tine et Elhaou ${ }^{6}$.

D'un point de vue empirique, ce travail sur l'(es) usage (s) des technologies en période de conflit en prenant comme étude de cas celui de Mango (au Togo en 2015) mobilise trois types de données. Premièrement, il se base sur une précédente étude réalisée sur la communication gouvernementale en période de crise au Togo (Yao, 2016). Il s'agissait de recueillir les articles de presse écrite (quotidien et hebdomadaire) publiés sur un certain nombre de crises/conflits (dont celui de Mango) afin d'analyser les pratiques communicationnelles gouvernementales durant ces périodes ${ }^{7}$. La lecture de ces articles de presse a servi de base pour cette recherche puisqu' elle a révélé les motifs de ce conflit d'une part, et la mobilisation des populations situées à deux endroits différents (Lomé et Mango) d'autre part. Deuxièmement, un entretien semi-directif (composé de vingt questions) a été réalisé auprès de dix jeunes âgés de 30 à $40 \mathrm{ans}^{8}$, résidant à Lomé et à Mango au cours du mois de juin 2016. Cette démarche visait à étayer les données 
recueillies à la suite de la lecture des articles publiés dans la presse écrite et approfondir notre recherche sur les rapports aux technologies, à leur usage et à leur impact, afin de mieux cerner la manière dont ces personnes narrent le conflit et le sens qu'elles donnent à leurs pratiques communicationnelles. Les données recueillies ont été traitées manuellement par le biais de l'analyse de contenu (sans faire de catégorisation) compte tenu de la taille de l'échantillon d'une part, et, des objectifs visés d'autre part. Troisièmement, des recherches sur internet ont conduit sur les sites de partage tels que Facebook et YouTube. Les productions existantes sur ces sites ont été dès lors consultées (toujours au cours du mois de juin 2016) pour enrichir la recherche et mieux saisir les différents usages. Cette étude sur l'usage des technologies en période de conflit au Togo en prenant pour exemple le cas de Mango demeure une modeste contribution à la recherche en SIC sur le continent.

\section{L'installation des aires protégées au Togo : un sujet conflictuel par nature?}

9 Avant d'aborder les questions liées aux usages des technologies d'information durant ce conflit, il est important de rappeler quelques éléments favorisant la compréhension du contexte et du conflit. Pour mieux comprendre l'opposition des populations riveraines et celles vivant à Lomé par rapport à la réinstallation de ces aires protégées, il nous faut revenir sur la définition des aires protégées dans un premier temps avant d'aborder les problématiques liées à leur installation et gestion au Togo dans un second temps.

10 Il existe une profusion des recherches sur la problématique des aires protégées en Afrique subsaharienne notamment en géographie (Fournier, Sinsin, Mensah, 2007). J.A. McNeely les définit comme: "n'importe quelle zone faisant l'objet d'un contrôle particulier sur le plan juridique et administratif ou pour des raisons de tradition, ainsi que de mesures d'aménagement visant à conserver certaines de ses caractéristiques, constitue par définition une aire protégée " (McNeely, 1994, p. 3). Retraçant un tant soit peu l'historique de leur implémentation, il rappelle qu'au départ c'étaient des zones dont, «seule une partie des multiples ressources pouvaient être exploitées» (op. cit.) avant de devenir progressivement "un endroit dont l'un des aspects avait une telle importance que la totalité de la zone devait être soustraite aux activités humaines » (Idem). Ce changement d'interprétation posera des difficultés dans la pratique puisque les populations n'y adhéreront pas facilement, car elles perçoivent ces politiques d'instauration des aires protégées comme une tentative de délocalisation voire de privation de terres exploitables. Abondant dans le même sens, Tchamie (1994), s'appesantira sur l'hostilité des populations locales à l'égard des aires protégées au Togo, car d'après lui, la destruction volontaire des ressources naturelles contenues dans ces aires (à laquelle se livrent ses populations) est la preuve de l'existence d'un "conflit " entre les programmes étatiques et les besoins des populations. Il poursuit son analyse en mentionnant qu'une politique ayant pour objectif la sauvegarde de ces zones et la gestion de leurs ressources naturelles doit tenir compte simultanément des intérêts de la population locale et de ceux de l'Etat (Tchamie, 1994, p. 24).

$11 \mathrm{Au}$ Togo, en 1954, le parc national de la Fosse aux lions (d'une superficie de 1650 ha), était une forêt classée. En 1981, toute la vallée de l'Oti (qui s'étend des limites nord et nord-est du parc national de la Kéran jusqu'à l'extrémité nord-est du pays) sera mise sous protection. Ce faisant, la réserve de faune de l'Oti-Mandouri dont les limites ont été 
plusieurs fois repoussées (148000 ha) sera créée (Tchamie, 1994). D'après l'auteur, l'installation et la gestion de cette zone protégée a causé "un tort immense » à la population, car les «objectifs politiques de la protection de l'environnement ont complètement occulté les raisons qui ont présidé à la création de zones protégées». (Tchamie, 1994, p. 24). Il cite Sournia (1990) pour qui, les aires protégées sont devenues « des garde-manger entourés par la faim » dérogeant ainsi à leur raison d'être à savoir : un moteur du développement économique de régions qui sont rurales à plus de $90 \%$ (op.cit).

Le contexte socio-politique des années 1990 marqué par le vent de la démocratie (Guèye, 2009) servira d'arrière-plan aux populations pour manifester leur hostilité (Tchamie, 1994). Dans cette perspective, le domaine forestier a été l'objet d'attaques diverses à savoir : les coupes anarchiques de bois, les défrichements illicites, la réinstallation illégale des villages autrefois déplacés et la destruction massive de la faune sauvage «Avec les troubles sociaux de 1990, les populations se sont livrées à une chasse effrénée pour se venger et non pour satisfaire leur besoin de gibier»(Tchamie, 1994 p. 24). Cet article mentionne donc l'hostilité des populations riveraines à l'égard de ces projets d'installation d'aires protégées à cause des litiges fonciers et des dérives liées à la mauvaise gestion. En 1999, le gouvernement togolais a initié un processus de réhabilitation et de gestion participative des aires protégées avec l'appui de certains partenaires. Le Projet Renforcement du rôle de conservation du système national des Aires Protégées au Togo (PRAPT) sera lancé les 29 et 30 mai 2012 à Lomég. D'un coût total de deux milliards de FCFA, il est financé par plusieurs partenaires dont le Programme des Nations Unies pour le Développement (PNUD) et l'Union Economique et Monétaire Ouest Africaine (UEMOA) ${ }^{10}$. C'est la mise en œuvre de ce projet dans la préfecture de l'Oti (complexe Oti - Kéran Mandouri) qui suscite l'opposition d'une partie de la population et notamment celle de Mango à cause des séquelles du passé comme le rappelle cet article publié dans l'hebdomadaire Golfe Info ${ }^{11}$ :

«Il faut dire que l'opposition des populations riveraines à ces aires protégées, ne date pas d'aujourd'hui. Elles qui se sont toujours senties lésées dans leurs activités quotidiennes, de culture, d'élevage, de pêche et de chasse, au bénéfice d'une population animale dont ils ne tirent aucun bénéfice. Mieux, elles jugent la protection de l'environnement dans leur zone, un handicap à leur développement socio-économique ».

13 Les propos de cet article de presse (sur lesquels toutes les publications consultées dans le cadre de cette étude ont insisté) viennent corroborer la publication du Professeur T. K. K. Tchamié datant de 1994. Ce conflit est donc révélateur de la difficulté des gouvernants à conduire un projet d'une telle envergure en impliquant et mobilisant toutes les couches de la population dans la perspective de la communication publique (Zémor, 2008). La naissance de ce conflit démontre la sensibilité du sujet liée à la réinstallation des aires protégées dans cette partie du Togo.

\section{La conduite du projet : entre sous-estimation de la mobilisation juvénile et déficit de communication}

Comme dit précédemment, le discours médiatique et la narration de ce conflit dans l'espace public ont fait apparaître plusieurs facteurs ayant concouru à son échec. Parmi ceux -ci nous retenons deux en particulier à savoir : la mobilisation et l'implication des jeunes natifs de la région résidant à Lomé et les populations riveraines; et le déficit de la communication autour du projet. La majorité des personnes qui se sont mobilisées et sont 
restées visibles dans l'espace public à travers les couvertures médiatiques était à majorité jeune (30-45ans en moyenne) comme le confirme les résultats de l'enquête menée ${ }^{12}$. Cette jeunesse, loin de représenter une population homogène, est constituée de personnes issues de catégories socioprofessionnelles différentes (jeunes cadres, étudiants, élèves, enseignants, etc.) voire de personnes sans emploi comme le montre cet extrait d'entretien : "Tout le monde se sent concerné par ce projet de faune; mais les jeunes sont plus visibles, car la majorité des jeunes de Mango sont peu instruits, beaucoup sont au chômage et vivent de l'agriculture. Ils perçoivent donc ce projet comme une menace ». Ils se mobilisent, car ils ont subi les méfaits de ces aires protégées durant leur enfance et ont vu leurs parents en pâtir. Ces jeunes ont un niveau d'instruction relativement élevé comparativement à celui de leurs parents des années 50 . De plus, leurs attentes concernant la conduite des projets et la gestion des politiques publiques sont plus fortes et nettement différentes de celles de leurs parents. Leurs expériences individuelles/ collectives, leur ouverture vers le monde extérieur (grâce en partie à "leur frère" de la diaspora) et la diffusion d'informations et d'images provenant d'horizons divers a permis à cette génération de prendre conscience de l'importance de sa participation au jeu social et d'intervenir sur le cours des évènements la concernant (Tine et Elhaou, 2015, p. 68). Les gouvernants dans leur conduite de ce projet ont omis ce changement de données en reconduisant les anciennes pratiques: appui sur les autorités locales (préfets, chefs traditionnels) et les "représentants/ aînés" de ces populations résidant à Lomé. Ce faisant, la force représentée par ces jeunes et leur capacité de mobilisation dans la durée a été minorée.

15 Au-delà de cette interprétation déduite suite à la collecte des données, le manque de communication a également joué un rôle clé dans la naissance de ce conflit.

La communication est un élément clé de la gouvernance démocratique, car elle se situe au cœur de la conduite des politiques publiques (Dubé, Facal, Bachir, 2009). Force est pourtant de constater que les gouvernants dans bon nombre de pays africains n'ont pas encore admis cette réalité (Toa, 2012). Le culte du secret et d'autres réalités d'ordre anthropologique et socioculturel entravent la mise en place d'une réelle politique de communication publique susceptible d'expliquer aux citoyens les grandes politiques nationales afin de susciter leur adhésion et implication (Zémor, 2008). Comme mentionné plus haut, les discours médiatiques ont également mis l'accent sur le déficit de la communication qui serait l'une des causes du conflit. Le quotidien Forum de la semaine, relate les propos du président du groupe parlementaire de la mouvance présidentielle qui "s'est insurgé contre le déficit de communication dont a souffert le projet et s'est félicité de la décision du chef de l'État de sursoir en attendant l'exécution du projet ${ }^{13} »$. Un député originaire de ladite préfecture mentionnera le déficit de communication, de sensibilisation et le refus d'une certaine partie de la population lors de l'intervention du ministre de l'Environnement et des ressources forestières ${ }^{14}$. En réponse à ces interpellations, le ministre dit « soupçonner des mains noires derrière ces manifestations » faisant ainsi allusion à d'éventuelles manipulations politiques ${ }^{15}$ même s'il déclare par ailleurs "s'étonner que la communication qui se fait autour du projet ne restitue pas la réalité des choses ${ }^{16}$. Ces différents propos démontrent que les pouvoirs politiques n'ont pas pris la mesure du changement social opéré auprès des citoyens. Ceux-ci, notamment les plus jeunes attendent plus d'informations/communication dans la perspective de construction du sens. La communication publique ne se résume pas uniquement à l'information; elle doit construire du sens afin de susciter l'adhésion de tous les citoyens dans une perspective 
inclusive à en croire les propos du président de l'Assemblée Nationale: «afin que les populations comprennent mieux ce qu'on leur propose, qu'on les associe activement à la gestion ${ }^{17}$ ».

17 La négligence voire la sous-estimation de la mobilisation des jeunes et de la communication fera naître des crises durant ce conflit.

\section{Du conflit à la crise : une gestion difficile}

Un conflit est souvent défini comme un «état de tensions résultant de l'antagonisme entre deux individus ou collectivités » (Géré, 2000, p. 5). Selon Antoine -Denis N’DiminaMougola, "le concept de conflit est par ailleurs, et par extension, synonyme de guerre" (N'Dimina-Mougala, 2007, 121). La signification du concept de crise a beaucoup évolué dans le temps (Traoré, 2016). Etant dans ses premières acceptions liées au monde médical, il renvoyait à la décision et au jugement. Dans sa signification contemporaine, la crise est considérée comme un événement inattendu survenant dans un espace-temps limité et qui vient rompre un équilibre existant (Bouilloud, 2012, p. 155). Ainsi, l'opposition d'une partie de la population contre l'installation des aires protégées fait naître un conflit bien avant le mois de novembre 2015. Les évènements du mois de novembre 2015 sont des crises inhérentes au conflit. Si les populations avaient auparavant manifesté leur refus de voir la réalisation de ce projet sur leur territoire, ce sont les évènements des 6, 7 et 26 novembre 2015 qui vont alerter l'opinion publique et révéler ainsi les violences langagières, symboliques et physiques de ce conflit. En effet, la manifestation (apparemment pacifique et sans autorisation du 6 novembre) des populations riveraines va se solder par deux morts tués par les forces de sécurité. Un article publié dans le quotidien national Togopresse mentionne les faits relatés par le Ministre en charge de la sécurité à propos des évènements du 7 novembre 2015.

\footnotetext{
«Les jeunes sont revenus à la charge avec une nouvelle manifestation qui a engendré trois morts causées par un véhicule blindé dont les freins ont lâché ${ }^{18}$ Le 26 novembre, «un regain de violences a occasionné la mort par lynchage du commissaire principal régional de Police de la Région des Savanes (...) ce dernier se serait rendu sur les lieux de manifestation pour d'abord négocier avec les manifestants qui n'auraient effectué aucune demande d'autorisation de l'organisation de la manifestation, quand il sera d'abord fauché par un projectile. Il sera dès lors pris à partie par ces manifestants surchauffés qui l'auraient lynché à mort » lit-on dans l'hebdomadaire Forum de la semaine ${ }^{19}$.
}

La médiatisation de ces évènements sur le plan national, international et la diffusion des images sur les différentes plateformes de partage (WhatsApp, YouTube, Facebook) a mis en évidence leurs violences langagières (injures des manifestants à l'égard des autorités), symboliques (diffusion de photos de corps des décédés par les balles et des blessés) et physique des forces de sécurité (répression des manifestants, courses poursuites, etc.). Face à cette situation, le gouvernement togolais tiendra dans la journée du samedi 7 novembre une réunion de crise, présidée par le Chef de l'État. Dans l'expectative d'apaiser les tensions, le Projet de réhabilitation des aires protégées dans la région sera suspendu, « en attendant de plus amples consultations» (Golfe Info du 9 novembre 2015). 


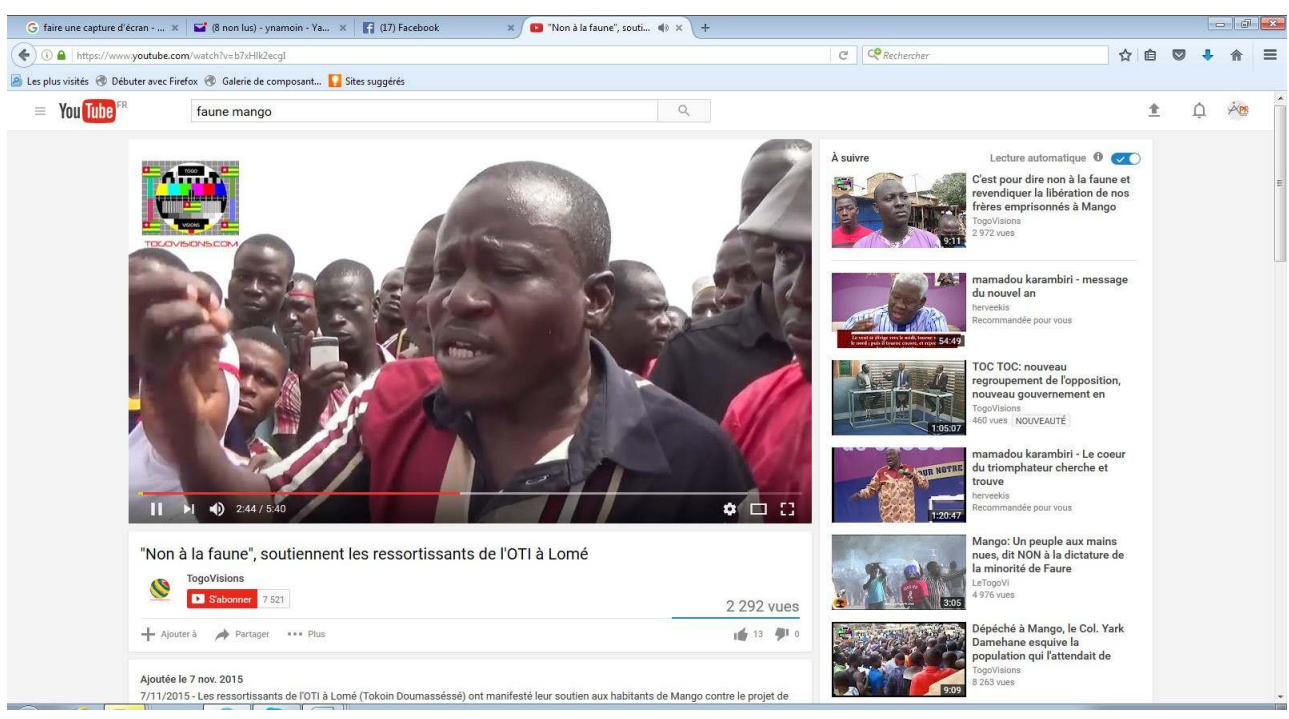

Dans la foulée et suite aux violences du 26 novembre (ayant entraîné la mort du commissaire), le gouvernement se réunit en conseil des ministres extraordinaire le lendemain et une série de décisions seront prises ${ }^{20}$. Elles seront diversement appréciées dans les commentaires sur les réseaux sociaux (Facebook) et les internautes rappellent le mot d'ordre "Non à la faune $»^{21}$. Le Togo a connu plusieurs crises de grandes ampleurs (incendie des deux grands marchés en janvier 2013, crise universitaire 2011, etc.). Néanmoins, il est à noter que c'est l'une des rares fois (durant ces cinq dernières années) où en l'espace de quelques jours, on assiste à une telle violence (décès des personnes, lynchage, injures, etc.) et à des décisions administratives d'une telle envergure: suspension du projet et nomination de nouvelles personnes. Voilà donc la manière dont le conflit sera parsemé de plusieurs crises notamment institutionnelles et révèlera la difficulté de la gestion d'une situation de crise comme celle-ci et le manque de communication durant celle-ci.

\section{Impact(s) de l'usage des technologies numériques durant le conflit}

Durant les moments forts de ce conflit, on a remarqué la prédominance du téléphone portable comme mode de partage d'informations. En effet, à cause de son accessibilité en termes de coût et des possibilités offertes aujourd'hui de produire et de diffuser du texte, de la voix et de l'image grâce notamment à l'application WhatsApp, cet outil demeure l'un des moyens par lesquels les populations échangent (Kiyindou, Anaté, Capo-Chichi, 2015). C'est également grâce aux SMS envoyés à plus de 2000 contacts que des centaines de personnes (originaires de la préfecture de l'Oti mais résidant à Lomé) se sont retrouvées pour manifester et proclamer leur soutien à leurs frères de Mango ${ }^{22}$. Toutefois, compte tenu du coût du sms, les têtes pensantes du mouvement à Lomé vont rapidement créer des groupes WhatsApp « afin de continuer à échanger sur le sujet à moindre coût pour trouver des solutions " (propos d'un enquêté). Les groupes suivants : Le vrai Mangolais, Oti News et Developpement, Anonche Ti Fanga (l'union fait la force en Tchokossi) et Kawansar demeurent actifs sur la plateforme WhatsApp et continuent à s'échanger des 
informations ${ }^{23}$. Les informations de toutes sortes sont échangées dans ces différents groupes et on note aussi des abonnés résidant à l'extérieur du Togo. En plus du téléphone, on note également la diffusion des vidéos sur le réseau de partage YouTube. Celles-ci montrent les grands épisodes du conflit: de la couverture des manifestations à Mango/ Lomé en passant par l'intervention des ministres de l'Environnement et de la sécurité devant les députés. La qualité de ces images est relativement bonne et elles sont presque toutes estampillées TogoVisions ${ }^{24}$. Ces images révèlent des manifestants en colère tenant des propos très virulents vis-à-vis des autorités administratives locales/ nationales. Elles sont vues par des milliers de personnes, mais très peu commentées par les internautes. Sur l'une des vidéos, l'une des personnes interrogées s'insurgeait contre les informations qualifiées de diffamatoires diffusées par la Télévision Nationale (TVT) au sujet des manifestations ${ }^{25}$. Sur Facebook, les recherches ont conduit à des pages comme : Non à la faune dans l'Oti, Témoignages sur la gestion de la Faune, Oti de Demain.

Ces pages demeurent des espaces où les internautes continuent de signifier leur refus de voir instaurer une faune sur leur territoire. Ces différents recours aux moyens technologiques pour produire et diffuser de l'information par les images, le texte et la voix, montrent le désir des populations d'informer elles-mêmes d'une part, et leurs compétences à l'utiliser, d'autre part. A ce propos, on note très peu de publications sur les blogs comparativement aux autres moyens susmentionnés. Ces usages couplés à la mobilisation des populations (à Mango et à Lomé) ont eu des impacts sur ce conflit eu égard aux décisions prises par les autorités politiques. Ils ont également insufflé une dynamique au cœur du conflit et ont amené les pouvoirs politiques à revoir leur agenda (en organisant des réunions de crise durant un week-end) et le projet (suspension jusqu'à nouvel ordre). Toutefois, l'usage des technologies et la mobilisation n'ont pas suffi pour résoudre définitivement ce conflit puisque les populations riveraines refusent d'enterrer les corps des personnes décédées maintenant ainsi, la pression sur les autorités politiques.

Cette recherche concernant l'usage des technologies numériques durant des périodes de crise/ conflit en prenant pour l'exemple le cas de la ville de Mango au Togo, ambitionnait d'analyser les pratiques communicationnelles et leur impact. Les résultats ont donc révélé l'impact de ces usages sur le conflit. Toutefois, celui-ci n'aurait pas été possible sans la mobilisation des populations concernées. Ainsi, nous nous éloignons des théories déterministes de l'usage des technologies numériques et postulons pour la primauté des logiques sociales. Dans ce cas, les frustrations/ hostilités des populations, le déficit d'une réelle politique de communication publique sont à la base du conflit et les technologies numériques ont servi à lui donner davantage de résonnance dans l'espace public. Ce faisant, les deux hypothèses de départ ont été confirmées. Hormis ces résultats, l'étude a également permis de mettre à jour certains dysfonctionnements institutionnels notamment en matière de communication publique. L'observation du déroulement $d u$ conflit et le discours médiatique ayant mis l'accent sur la mobilisation « des filles et fils du milieu », on peut s'interroger à la suite des travaux réalisés sur l'ethnicité et les crises (Kouméalo Assima-Kpatcha- Tsigbé, 2016) sur la part de la dimension ethnique dans la résolution des conflits et crises dans ce pays. 


\section{BIBLIOGRAPHIE}

Kouméalo Anate et al. (dir.), Ethnicité, crises sociopolitiques et processus de réconciliation nationale, Letria, Lomé, 2016.

Jean-Philippe Bouilloud, De la « crisologie » à la « risquologie », Communications, 2012/2 (n 91), pp. 153-159.

Rémy Le Champion, Journalisme 2.0, La documentation française, Paris, 2012.

Anne Fournier, Brice Sinsin, Guy Appolinaire Mensah, (dir.), Quelles aires protégées pour l'Afrique de l'Ouest? IRD Editions, Marseille, 2007.

Marie-Soleil Frère (dir.), Médias et Conflits, vecteurs de guerre ou acteurs de paix, Edition GRIP, Bruxelles, 2005.

François Géré (dir.), Dictionnaire de la pensée stratégique, Larousse-Bordas, Paris, 2000.

Babacar Guèye, «La démocratie en Afrique : succès et résistances ", Pouvoirs, 2009/2 (n 129), pp. 5-26.

Sarah Joseph, « Social Média, Human Rights and Political Change », Boston College International \& Comparative Law Review, Vol 35, 2011, pp. 145-188, disponible en ligne : http:// lawdigitalcommons.bc.edu/cgi/viewcontent.cgi ?article =1667\&context =iclr, consulté le 4 juillet 2016.

Alain Kiyindou et al. (dir.), Quand l'Afrique réinvente la téléphonie mobile, L'Harmattan, Paris 2015.

Antoine-Denis N’Dimina - Mougala, « Les conflits africains au XXè siècle. Essai de typologie ", Guerres mondiales et conflits contemporains, 2007/1 ( $\left.n^{\circ} 225\right)$, p. 121- 131. Disponible en ligne : https://www.cairn.info/revue-guerres-mondiales-et-conflits-contemporains-2007-1page-121.htm, consulté le 15 juillet 2016.

Jeffrey A, MCNeely, Parcs et aires protégées, Editorial, Unasylva n 176, 1994.

Serge Proulx, « Penser les usages des technologies de l'information et de la communication aujourd'hui : enjeux- modèles - tendances, in Enjeux et Usages des TIC : aspects sociaux et culturels, Lise Vieira et Nathalie Pinède (dir.) Tome 1, PUB, 2005, pp. 7-20.

Alexandre Serres, « L'évaluation de l'information à l'heure du web 2.0 : entre changement et continuité », Journée d'Etude Médiadix, Urfist Paris, 2010.

Thiou Tanzidani Komlan Tchamie, Enseignements à tirer de l'hostilité des populations à l'égard des aires protégées au Togo, Unasylva n 176, 1994, pp. 22- 27.

Benoît Tine et Mohamed -Ali Elhaou, « Mouvements populaires en Afrique, primauté technologique ou antériorité sociale ? Étude de cas du Sénégal et de la Tunisie, Communication, Technologie et Développement, $\mathrm{n}^{\circ}$ 2, octobre 2015, pp. 64-74. Disponible en ligne : http:// www.comtecdev.com/fr/telecharger/Tine_Elhaou.pdf, consulté le 10 juin 2016.

Jules Evariste Toa, « Communication et Culte du secret dans les organisations en Côte d'Ivoire », Congrès de la SFSIC, Rennes juin 2012. 
Oumar Cheick Traoré, Les enjeux de la communication gouvernementale en période de crise. Le cas du Mali, Communication, Colloque International Bamako 2016, Université des Lettres et des Sciences Humaines de Bamako, Bamako, 11 mai 2016.

Namoin Yao, Une analyse de la communication gouvernementale en période de crise, Communication, Colloque International Bamako 2016, Université des Lettres et des Sciences Humaines de Bamako, Bamako, 11 mai 2016.

Pierre Zémor, La communication publique, Paris, Que sais-je ? 2008.

\section{NOTES}

1. Ici nous faisons allusion au fait que les logiques médiatiques, les lignes éditoriales et l'agenda setting peuvent occulter certains sujets.

2. Mango est une ville située à $550 \mathrm{~km}$ à peu près de Lomé, la capitale du Togo. Elle est dans la préfecture de l'Oti (la région de la Savane) au nord du pays.

3. Nous avons retenu la presse écrite parce que le matériau est facile à recueillir. Les reportages télévisuels auraient nécessité des courriers de demande et des autorisations de chaînes pour accéder aux enregistrements.

4. Les différents reportages diffusés sur les chaînes nationales (TVT) et privées (LCF en occurrence) ont mis l'accent sur les différentes marches de protestations organisées par les jeunes natifs de la région résidant à Lomé au cours du mois de novembre 2015.

5. Au moment où cet article était en cours de rédaction (juillet 2016), le conflit était toujours latent.

6. Dans leur article sur «Mouvements populaires en Afrique, primauté technologique ou antériorité sociale? Étude de cas du Sénégal et de la Tunisie » (voir référence complète dans la bibliographie), Benoit Tine et Mohamed -Ali Elhaou reprennent les travaux de Sarah Joseph "Social Média, Human Rights and Political Change ", pour qui les logiques sociales se situeraient à l'avant-garde d'un désenchantement citoyen. Dans cette perspective, « les supports numériques ne sont qu'une alternative faible et provisoire favorisant la mise en scène d'un esprit vindicatif spectaculaire sans plus» (Tine \& Elhaou:2015, 66). Pour eux, "les mouvements sociaux traduisent une frustration sociale de longue durée et n'ont pas attendu les médias numériques (Facebook ou Twitter) pour prendre forme » (Tine \& Elhaou : 2015, 67)

7. Dans le cadre de cette recherche sur la communication gouvernementale en période de crise, 286 articles publiés par cinq supports de presse écrite (quotidien/hebdomadaire et bihebdomadaire) et portant sur quatre crises ont été recueillis.

8. Au total, 21 articles ont été rassemblés dans quatre (4) publications de presse écrite essentiellement des quotidiens et des hebdomadaires. Il s'agit de : Liberté (quotidien privé (10)), Golfe Infos (bihebdomadaire privé (01)), Forum de la semaine (hebdomadaire privé (09), Togopresse (quotidien national (01)).

9. http://www.panapress.com/Le-Togo-va-restaurer-ses-aires-protegees-selon-les-standardsinternationaux--13-830406-18-lang1-index.html. consulté le 6 juillet 2016.

10. Golfe Info du 9 novembre 2015.

11. Les personnes ayant participé à l'étude précisent que fondamentalement tout le monde est concerné ; même si ce sont les jeunes qui se mobilisent davantage et organisent les marches de protestation.

12. Forum de la Semaine $\mathrm{n}^{\circ} 2008$ du 11 novembre 2015).

13. Voir vidéo Youube. https://www.YouTube.com/watch?v=j-RA-tgwmo8. consulté le 10 juin 2016.

14. Forum de la Semaine $\mathrm{N}^{\circ} 2007 \mathrm{du} 10$ novembre 2015. 
15. Forum de la Semaine $\mathrm{n}^{\circ} 2008$ du 11 novembre 2015.

16. Forum de la Semaine $n^{\circ} 2008$ du 11 novembre 2015.

17. Propos du Président de l'Assemblée Nationale lors du passage des ministres de l'environnement et de la sécurité. Togopresse $\mathrm{N}^{\circ} 9662$ du 12 novembre 2015.

18. Togopresse $n^{\circ} 9662$ du 12 novembre 2015

19. Forum de la Semaine $n^{\circ} 2019$ du 27 novembre 2015.

20. Il s'agira entre autres de la nomination d'un nouveau Préfet, l'installation sans délai du nouveau chef de canton de Mango, le repli des éléments de force de 3eme catégorie (militaires) initialement réquisitionnés par l'autorité compétente locale et la poursuite diligente de la procédure judiciaire. Rappelons que le départ du Préfet en poste lors de la crise a été réclamé par les manifestants.

21. Voir page Facebook «Oti de Demain » et le titre des vidéos postées sur YouTube.

22. Lors de notre enquête nous avons rencontré la personne à la base de cette initiative. Un jeune architecte, originaire de Mango offrant des services de messagerie aux organisations au Togo et détenant d'après ses propres propos une base de données contenant les contacts téléphoniques des personnes originaires de cette région.

23. Dans le cadre de cette recherche, nous sommes inscrits dans le groupe "Oti et Développement » (WahtsApp) et « Non à la faune dans l'Oti » (groupe Facebook).

24. www.togovisions.net

25. Voir Vidéo sur YouTube : http://www.youtube.com/watch?v=b7xHlk2ecgI consulté le 27 juin 2016

\section{RÉSUMÉS}

La ville de Mango située dans la région septentrionale du Togo a été le théâtre d'une crise dont les médias ont largement fait écho au mois de novembre 2015. Au cours de ces évènements, on a observé une grande mobilisation des populations riveraines et des natifs de cette localité résidant à Lomé. Cet article s'intéresse à la manière dont ils ont interagi avec ces différents outils (internet et téléphonie mobile), les finalités et l'impact de ces usages dans la résolution de cette crise. L'analyse de ces évènements à travers la couverture médiatique de la presse écrite et les différents outils ont révélé des déficits communicationnels au niveau de la conduite de ce projet. Elle a surtout permis d'inscrire les usages dans un contexte social caractérisé par des pesanteurs politiques déterminant la résolution de ce conflit.

Mango, a city located in the northern region of Togo, was the scene of a crisis that was widely reported in November 2015 news media. During these events, there was a great mobilization of the neighboring populations and the natives of that locality residing in Lomé. This article examines how they interacted with these different tools (internet and mobile telephony), the purposes and impact of these uses in solving that crisis. The analysis of these events through media coverage of the print press, and the various tools revealed communication deficits in the carrying out of this project. Above all, it has made it possible to put customs into a social context characterized by political constraints determining the resolution of this conflict.

La ciudad de Mango situada en la región septentrional de Togo fue el escenario de una crisis, los medios de comunicación ampliamente reportados en noviembre de 2015. Durante estos eventos, 
hubo una gran movilización de los jóvenes nativos y el uso intensivo de las tecnologías de la información y la comunicación. Este artículo analiza cómo han interactuado con estas herramientas, los resultados y el impacto de estos usos en la solución de esta crisis. El análisis de estos eventos a través de la cobertura mediática y las diversas herramientas ha permitido centrarse en su aspecto generacional y étnico. Especialmente ha permitido centrarse en esos usos en un contexto social caracterizado por consideraciones políticas para resolver este conflicto.

INDEX

Palabras claves : Mango, crisis, movilización juvenil, usos tecnológicos, etnia

Keywords : Mango, crisis, juvenile mobilization, technological uses, ethnicity

Mots-clés : Mango, crise, jeunes, usages technologiques, ethnicité

\section{AUTEUR}

\section{NAMOIN YAO}

Université de Lomé 